\title{
A REMARK ON OPERATING GROUPS
}

\author{
YANMING WANG \\ Department of Mathematics \\ Zhongshan University \\ Guangzhou 51027, P. R. China \\ School of Mathematics \\ University of Minnesota \\ Minneapolis, MN 55455 USA \\ (Received October 6, 1994 and in revised form January 5, 1995)

\begin{abstract}
Let $G$ be a finite group and $H$ be an operator group of $G$. In this short note, we show a relationship between subnormal subgroup chains and $H$-invariant subgroup chains. We remark that the structure of $H$ is quite restricted when $G$ has a special $H$-invariant subgroup chain.
\end{abstract}

KEY WORDS AND PHRASES. Solvable groups, supersolvable groups, operator groups.

1991 AMS SUBJECT CLASSIFICATION CODES 20010, $200 D 15$.

There are many properties in group theory which are defined in terms of some subgroup chain of a group. For example, the Jordan-Holder Theorem says that any two composition series of a finite group are equivalent. A group $G$ is solvable if $G$ has a subnormal subgroup chain with cyclic factors. A finite group $G$ is supersolvable if and only if $G$ has a normal subgroup chain with cyclic factors. When we consider a group $H$ acting on a group $G$, we are very interested in $H$-invariant subgroup chains of $G$. If abstract conditions are imposed on $H$-invariant chains of subgroups of $G$, then it is not surprising if there are some restrictions on the structures of both $G$ and $H$. There are a lot of results about the structure of $G$. For example, consider $H=G$ acting on $G$ by conjugation. Then $G$ is supersolvable if and only if $G$ has an $H$-invariant subgroup chain with cyclic factors. $G$ is solvable if and only if $G$ has an $H$-invariant subgroup chain with abelian factors. However, there is not much research on the structure of $\boldsymbol{H}$. In this short note, we remark on the structure of the operator group.

Our notation follows [R]. Let $G$ be a group. A subgroup chain of $G$ is a finite sequence $\left(G_{0}, G_{1}, \cdots, G_{n}\right)$ of subgroups of $G$ such that $1=G_{0}<G_{1}<\cdots<G_{n-1}<G_{n}=G$.

Lemma 1. Let $G$ be a group and $H$ be a group acting on $G$. Let $M, N$ be two H-invariant subgroups of $G$ with $M<N$. Denote $C_{H}(N: M)=\left\{h \in H: \forall x \in N, x^{h} M=x M\right\}$. Then $C_{H}(N: M)$ is a normal subgroup of $H$.

Proof. $H$ acts on the set $\{x M \mid x \in N\} . C_{H}(N: M)$ is the kernel of this action.

As the main theorem, we prove the following

Theorem 2. Suppose the group $H$ acts on the group $G$. Suppose that $G_{1} \triangleleft G_{2} \triangleleft \cdots \triangleleft G_{n-1} \triangleleft$ $G_{n} \triangleleft G$ is a partial $H$-invariant subgroup chain with cyclic factors. Then $H / C_{H}\left(G: G_{1}\right)$ is supersolvable. 
Proof. We prove the theorem by induction on $n$. Suppose $n=1$ so that $G / G_{1}$ is cyclic. $H / C_{H}\left(G: G_{1}\right)$ is isomorphic to a subgroup of, the abelian group, $\operatorname{Aut}\left(G / G_{1}\right)$, and hence $H / C_{H}\left(G: G_{1}\right)$ is supersolvable. Now assume that $n>1$. Consider $H$ acting on both the chain $G_{1} \triangleleft G_{2} \cdots \triangleleft G_{n}$ and the chain $G_{2} \triangleleft \cdots \triangleleft G$. By induction, we have that both $H / C_{H}\left(G: G_{2}\right)$ and $H / C_{H}\left(G_{n}: G_{1}\right)$ are supersolvable. Let $M=C_{H}\left(G: G_{2}\right) \cap C_{H}\left(G_{n}: G_{1}\right)$ and $N=M C_{H}(G$ : $\left.G_{1}\right)$. Then $H / M$ is supersolvable and so is $H / N$.

We prove that $N / C_{H}\left(G: G_{1}\right)$ is a cyclic group.

Let $G / G_{n}=\left\langle g G_{n}\right\rangle$. For any element $a \in N$, by definition, we have that $g^{a}=g g_{a}$ with $g_{a} \in G_{2}$. Define $\phi: N \rightarrow G_{2} / G_{1}$ as follows: $\phi: a \rightarrow g_{a} G_{1}$. It is easy to prove that $\phi$ is well defined and $\phi$ is a homomorphism. In fact, for $a_{1}, a_{2} \in N$, we have that $\phi\left(a_{1}\right)=$ $g_{a_{1}} G_{1} . \phi\left(a_{2}\right)=g_{a_{2}} G_{1}$. Note that $g^{a_{1} a_{2}}=\left(g^{a_{1}}\right)^{a_{2}}=\left(g g_{a_{1}}\right)^{a_{2}}=g^{a_{2}}\left(g_{a_{1}}\right)^{a_{2}}=g g_{a_{2}}\left(g_{a_{1}}\right)^{a_{2}}$ and $g_{a_{2}}\left(g_{a_{1}}\right)^{a_{2}} \in G_{2}$. So by definition of $\phi$, we obtain that $\phi\left(a_{1} a_{2}\right)=g_{a_{2}}\left(g_{a_{1}}\right)^{a_{2}} G_{1}$. Notice that $a_{2} \in N$ and $g_{a_{1}} \in G_{2} \leq G_{n}$. We now have that $\left(g_{a_{1}}\right)^{a_{2}} G_{1}=g_{a_{1}} G_{1}$. Since $\phi\left(a_{1} a_{2}\right)=$ $g_{a_{2}} g_{a_{1}} G_{1}=g_{a_{1}} G_{1} g_{a_{2}} G_{1}=\phi\left(a_{1}\right) \phi\left(a_{2}\right), \phi$ is a homomorphism from $N$ to $G_{2} / G_{1}$. It is clear that $C_{H}\left(G: G_{1}\right) \leq K \operatorname{er} \phi$. We claim that $K \operatorname{er} \phi=C_{H}\left(G: G_{1}\right)$. Since $G / G_{n}=<g G_{n}>$, for any element $x \in G$, we have that $x=g^{k} g_{n}$ for some $k$ and with $g_{n} \in G_{n}$. If $a \in k e r \phi$, then $\phi(a)=g_{a} G_{1}=G_{1}$ with $g^{a}=g g_{a}$. This implies that $g^{-1} g^{a} \in G_{1}$. Now $\left(g^{a}\right) G_{1}=g G_{1}$ and $\left(g_{n}^{a}\right) G_{1}=\left(g_{n}\right) G_{1}$ yields that $\left(x^{a}\right) G_{1}=x G_{1}$ for every $x \in G$, hence $a \in C_{H}\left(G: G_{1}\right)$. This shows that $\operatorname{Ker} \phi=C_{H}\left(G: G_{1}\right) ; N / C_{H}\left(G: G_{1}\right)$ isomorphic to a subgroup of the cyclic group $G_{2} / G_{1}$, and so $N / C_{H}\left(G: G_{1}\right)$ is cyclic.

Since $H / N \cong\left(H / C_{H}\left(G: G_{1}\right)\right) /\left(N / C_{H}\left(G: G_{1}\right)\right)$ is supersolvable and $N / C_{H}\left(G: G_{1}\right)$ is cyclic, we have that $H / C_{H}\left(G: G_{1}\right)$ is supersolvable.

R. Baer investigated the supersolvable immersion and proved the following theorem.

Baer's Theorem. ([H] p. 119 Hilfssatz 9.8). Let $X$ be a group. Suppose that $1=G_{1} \triangleleft$ $G_{2} \triangleleft \cdots \triangleleft G_{n-1} \triangleleft G_{n}$, with each $G_{i} \triangleleft X$ such that $\left|G_{1+1} / G_{i}\right|$ are primes. Then $X / C_{X}\left(G_{n}\right)$ is supersolvable.

Remark: Let $H=X$ acts on $1=G_{1} \triangleleft G_{2} \triangleleft \cdots \triangleleft G_{n-1} \triangleleft G_{n}$ by conjugation. Let $G_{n}$ be the $G$ in Theorem 2. Let 1 be the $G_{1}$ in Theorem 2. Then $C_{H}\left(G: G_{1}\right)$ now is $\left\{x \in G \mid y^{-1} y^{x}=\right.$ $\left.1, \forall y \in G_{n}\right\}$, and so it is $C_{G}\left(G_{n}\right)$. Then this implies Baer's Theorem.

Corollary 3. Let $G$ be a group and $H$ be an automorphism group of $G$. Suppose that $1 \triangleleft$ $G_{1} \triangleleft G_{2} \triangleleft \cdots \triangleleft G_{n} \triangleleft G$ is a finite cyclic $B$-invariant subnormal subgroup chain. Then $H$ is supersolvable.

Proof. Notice that $C_{H}(G: 1)=1$ in this case, Theorem 2 implies the corollary.

We know that a group $G$ is solvable if and only if $G$ has a subnormal cyclic subgroup chain which can be refined to a subnormal subgroup chain, where each of the factors is of prime order. When we consider a group $H$ acts on a group $G$, it is hard to say whether we can find a $H$-invariant subgroup chain with some particular property. In fact, it is not true in general for some properties, the structure of $\boldsymbol{H}$ is quite restricted as we showed in Corollary 5 . Conversely, if we know that there exists a $H$-invariant subgroup chain, usually we don't know whether we can find a subnormal subgroup chain with the same property. However, we can say a little more for a finite solvable group.

Lemma 4. Let $G$ be a finite solvable group and $H$ be a group acting on $G$. Suppose that there exists a $H$-invariant subgroup chain of $G, 1=G_{1}<G_{2}<\cdots<G_{n-1}<G_{n}=G$, with $\left|G_{i+1}: G_{i}\right|$ a prime for $i=1,2, \cdots, n-1$. Then there exists a subnormal subgroup chain of $G$ with the same property.

Proof. We prove it by induction on $|G|$. If $G$ is abelian, then every subgroup chain is a subnormal subgroup chain, and we are done. Suppose that $G$ is not abelian. Since $G$ is solvable, there exists a minimal characteristic subgroup $N$ of $G . N$ is $H$-invariant and $N$ is an elementary abelian $p$-group for a prime $p \in \pi(G) .\left|G_{i+1} N / N: G_{i} N / N\right||| G_{i+1}: G_{i} \mid$ and so it is 1 or a prime. $1=N / N \leq G_{2} N / N \leq \cdots \leq G_{n-1} N / N \leq G / N$ is a $H$-invariant chain. Delete the repeated term in the chain. We get a chain satisfying the hypotheses of the Lemma. By induction, we can prove that there exists a subnormal $H$-invariant subgroup chain of $G / N$ satisfying the hypotheses of the Lemma, hence we have $N=N_{1} \triangleleft N_{2} \triangleleft \cdots \triangleleft N_{m-1} \triangleleft N_{m}=G$; 
where $N_{i}$ is $H$-invariant, and $\left|N_{i+1}: N_{i}\right|$ is a prime. It is sufficient to prove that $N$ satisfies the hypotheses of the Lemma. Consider $M_{i}=N \cap G_{i} . M_{i}$ is a $H$-invariant subgroup of $N$. We have the following $H$-invariant subgroup chain of $N$ :

$$
1=M_{1} \leq M_{2} \leq \cdots \leq M_{n-1} \leq M_{n}=N
$$

We only need to prove that $\left|M_{i+1}: M_{i}\right|=p_{i}$ is either 1 or prime. Note that $\left|N G_{i+1}: N G_{i}\right|$ $=\left(\left|G_{i+1}: M_{i+1}\right|\right) /\left(\left|G_{i}: M_{i}\right|\right)=\left(G_{i+1}: G_{i} \mid\right) /\left(\left|M_{i+1}: M_{i}\right|\right)$. Therefore $\left|M_{i+1}: M_{i}\right|\left|G_{i+1}: G_{i}\right|$ and hence it is 1 or prime. Delete the repeated term in the chain. We get the required subgroup chain of $N$. Since $N$ is abelian, the chain is a subnormal chain; this completes the proof of the Lemma.

Combining Theorem 2 and Lemma 4, we get the following Corollary 5.

Corollary 5. . Let $G$ be a finite solvable group and $H$ be a group acting on $G$. Suppose there exists a $H$-invariant subgroup chain of $G, 1=G_{1}<G_{2}<\cdots<G_{n-1}<G_{n}=G$, with $\left|G_{i+1}: G_{i}\right|$ a prime for $i=1,2, \cdots, n-1$. Then $H / C_{H}(G)$ is supersolvable.

Proof. It is clear if we note that $C_{H}(G)=C_{H}(G: 1)$.

ACKNOWLEDGEMENT. This work was partially supported by National Science Foundation of China. The author is grateful to professor Thomas Berger for his help. The author would like to thank the referee for his/her comments.

\section{References}

[H]. B. Huppert, Endliche Gruppen. Springer-Velag, New York, Bonn, 1967.

[R]. D.R.S. Robinson, A Course of Group Theory. Springer-Verlag, New York, Bonn, 1982. 


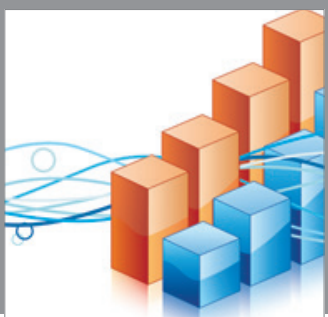

Advances in

Operations Research

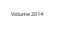

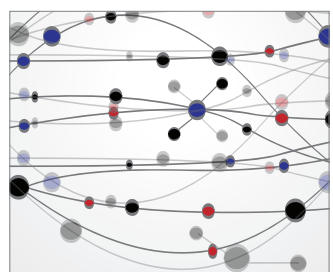

\section{The Scientific} World Journal
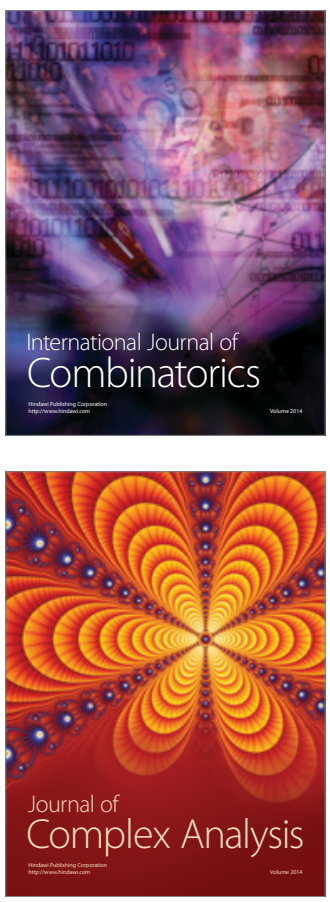

International Journal of

Mathematics and

Mathematical

Sciences
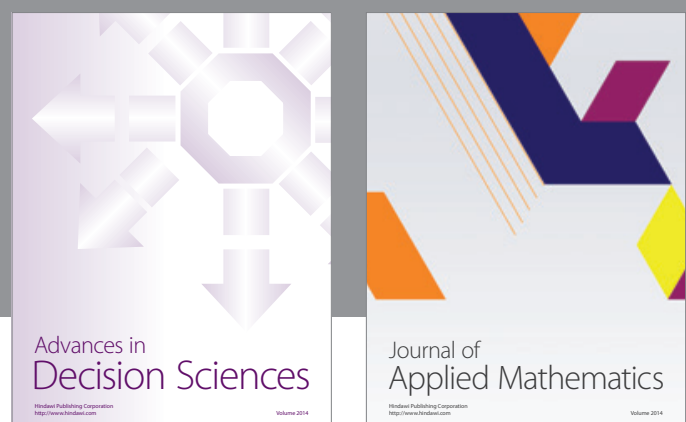

Journal of

Applied Mathematics
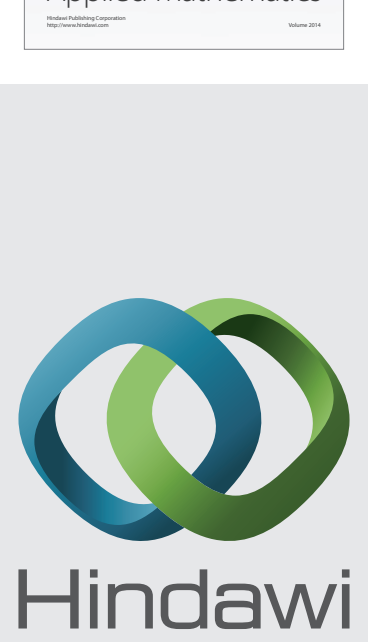

Submit your manuscripts at http://www.hindawi.com
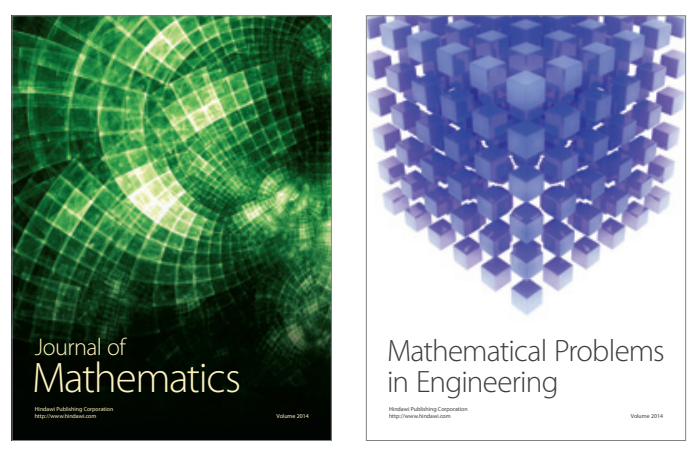

Mathematical Problems in Engineering
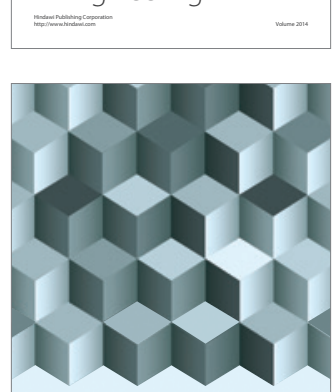

Journal of

Function Spaces
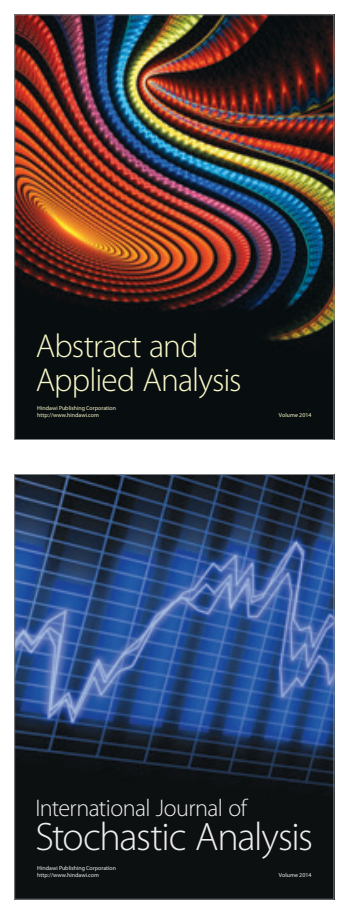

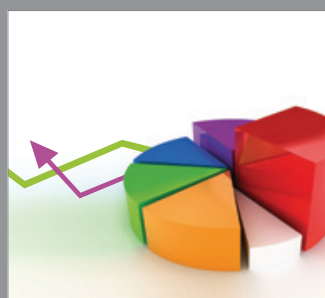

ournal of

Probability and Statistics

Promensencen
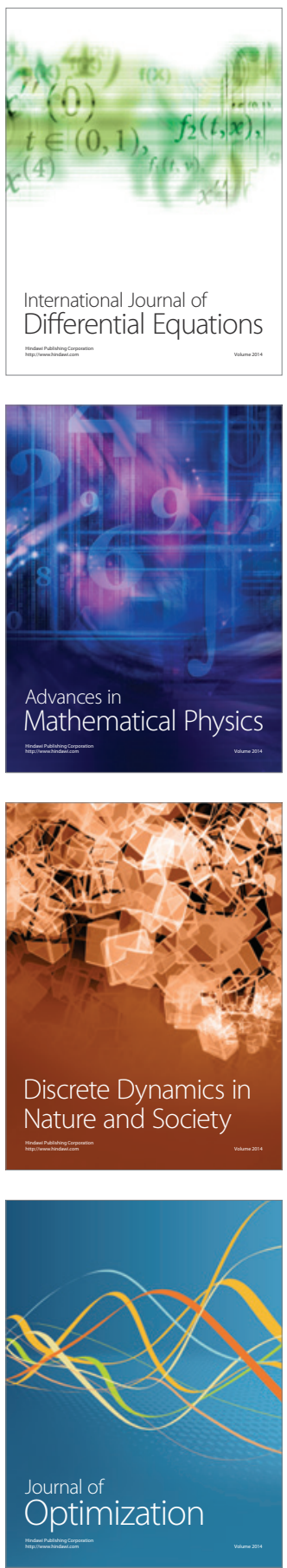